\title{
Orbit and spin evolution of the synchronous binary stars on the main sequence phase
}

\author{
Lin-Sen Li \\ School of physics, Northeast Normal University, Changchun 130024, China; lils653@nenu.edu.cn
}

Received 2010 August 20; accepted 2012 May 17

\begin{abstract}
The sets of the synchronous equations are derived from the sets of nonsynchronous equations The analytical solutions are given by solving the set of differential equations. The results of the evolutionary tendency of the orbit-spin are that the semimajor axis shrinks gradually with time: the orbital eccentricity dereacses gradually with time until the orbital circularization; the orbital period shortens gradually with time and the rotational angular velocity of primary component speed up with time gradually before the orbit-rotation achieved the circularization The theoretical results are applied to evolution of the orbit and spin of synchronous binary stars Algol A, B on the main sequence phase The circularization time and life time (age) and the evolutional numerical solutions of orbit and spin when circularization time are estimeted for Algol A, B. The results are discussed and concluded.
\end{abstract}

Key words: Binaries: close — rotation — evolution

\section{INTRODUCTION}

The tidal friction plays an important role in the evolution of the orbit and spin of close binary system. Earliest, the author researching this topic is Zahn (1965, 1966a,b, c, 1975). Alexander (1973) firstly studied the dynamical problem of the tidal friction in close binary system by using the method employed by Darwin (1879). Later on, Hut (1980, 1981) generalized the method given by Alexander (1973). He studied the stability of tidal equilibrium and tidal evolution in close binary system by using the method of energy and angular momentum. But their research dealt with a few synchronization. The sequential research for the synchronization of rotation are given by Zahn $(1977,1978)$. Rajamohan \& Venkatakrishnan (1981) ever studied synchronization in binary stars. Giuricin et al. (1984a) researched synchronization in eclipsing binary stars and Giuricin et al. (1984b) also researched synchronization in early-type spectroscopic binary stars, Zahn \& Bouchet (1989) studied mainly the orbital circularization of late-type binary stars on the pre-main sequence and the theoretical results are given based on Zahn (1989). Pan (1996) calculated the circularization time scale by using the two mechanisms: one is the equilibrium tidal mechanism given by Zahn (1977), another is purely hydrodynamic mechanissm given by Tassoul (1987). Keppens et al. (2000) studied the rotational evolution of binary stars system: synchronization and circularization. Huang \& Zeng (2000) also researched evolution of non-synchronized binary stars with 9 solar mass and 6 solar mass. Meibom et al. (2005) studied obseration tidal synchronization in detached solar-type binary stars and Meibom et al. (2006) also researched an observational study of tidal synchronization in solar-type binary starss in open clusters M35 and M34. Although the author Li (1998, 2004, 2009) studied some methods for judging the synchronization of rotation of binary stars, but he does not studied the evolution of orbit-rotation of synchronous binary 
stars. In the present paper the author examined the evolutional tendency of orbit and spin of synchronous binary stars on the main sequence phase.

\section{THE EVOLUTIONAL EQUATIONS OF SYNCHRONOUS BINARY STARS DUE TO THE TIDAL FRICTION ON THE MAIN SEQUENCE}

The secular evolutional equations of the semi-major axis, $a$, eccentricity, $e$, and the rotational angular velocity, $\Omega$, due to tidal frication for non-synchronous binary stars are given by Zahn (1989).

$$
\begin{gathered}
\frac{1}{a} \frac{d a}{d t}=-\frac{12}{t_{f}} q(1+q)\left(\frac{R}{a}\right)^{8}\left\{\lambda^{22}\left(1-\frac{\Omega}{\omega}\right)\right. \\
\left.+e^{2}\left[\frac{3}{8} \lambda^{10}+\frac{1}{16} \lambda^{12}\left(1-2 \frac{\Omega}{\omega}\right)-5 \lambda^{22}\left(1-\frac{\Omega}{\omega}\right)+\frac{147}{16} \lambda^{32}\left(3-2 \frac{\Omega}{\omega}\right)\right]\right\} \\
\frac{1}{e} \frac{d e}{d t}=-\frac{3}{t_{f}} q(1+q)\left(\frac{R}{a}\right)^{8}\left[\frac{3}{4} \lambda^{10}-\frac{1}{8} \lambda^{12}\left(1-2 \frac{\Omega}{\omega}\right)\right. \\
\left.-\lambda^{22}\left(1-\frac{\Omega}{\omega}\right)+\frac{49}{8} \lambda^{32}\left(3-2 \frac{\Omega}{\omega}\right)\right] \\
\frac{d}{d t}(I \Omega)= \\
-\frac{6}{t_{f}} q^{2} M R^{2}\left(\frac{R}{a}\right)^{6}\left\{\lambda^{22}(\omega-\Omega)+e^{2}\left[\frac{1}{8} \lambda^{12}(\omega-2 \Omega)\right)\right. \\
\left.\left.-5 \lambda^{22}(\omega-\Omega)+\frac{49}{8} \lambda^{32}(3 \omega-2 \Omega)\right]\right\}
\end{gathered}
$$

Where $M$ and $R$ denote the mass and the radius of primary star, $q=\frac{M^{\prime}}{M}, M^{\prime}$ denotes the mass of the secondary star, $\omega$ denotes the orbital angular velocity (mean motion), $I$ denotes momentum of inertia, the convective friction time and $\lambda^{l m}$ are given by (Zahn \& Bouchet 1989)

$$
t_{f}=\left[\frac{M R^{2}}{L}\right]^{1 / 3}, \quad \lambda^{l m}=\lambda_{2}[2 \pi / 1 l \omega-m \Omega 1] .
$$

Here $L$ denotes the luminosity of the primary star.

Next, one derives the evolutional equations of synchronous binary stars. Zahn \& Bouchet (1989) point out that when the two components rotate with the orbital motion in synchronism: $|l \omega-m \Omega|=\omega$, then, all tidal coefficients are identical $\lambda^{l m}=\lambda$ except for $\lambda^{22}$. Hence in Equations (1) $-(3) \lambda^{11}=$ $\lambda^{10}=\lambda^{12}=\lambda^{32}=\lambda, \lambda^{22} \neq \lambda$. When we consider the two components rotate in synchronism, $\Omega=\omega$ or $\frac{\Omega}{\omega}=1$. Substituting these conditions into the Equations (1) - (3), the secular Equations (1) - (3) are reduced to the following simplified secular synchronized equations

$$
\begin{gathered}
\frac{1}{a} \frac{d a}{d t}=-114 q(1+q) \frac{\lambda}{t_{f}} e^{2}\left(\frac{R}{a}\right)^{8}, \\
\frac{1}{e} \frac{d e}{d t}=-21 q(1+q) \frac{\lambda}{t_{f}}\left(\frac{R}{a}\right)^{8}, \\
\frac{1}{\Omega} \frac{d \Omega}{d t}=36 q^{2}\left(\frac{M R^{2}}{I}\right) \frac{\lambda}{t_{f}} e^{2}\left(\frac{R}{a}\right)^{6}=36 q^{2}\left(\frac{M}{I}\right) \frac{\lambda}{t_{f}} e^{2} a^{2}\left(\frac{R}{a}\right)^{8} .
\end{gathered}
$$

We may also write down a supplementary secular equations according to keplerian third law

$$
\frac{1}{\omega} \frac{d \omega}{d t}=-\frac{3}{2} \frac{1}{a} \frac{d a}{d t}
$$




$$
\begin{gathered}
\frac{1}{P_{\text {orb }}} \frac{d P_{\text {orb }}}{d t}=\frac{3}{2} \frac{1}{a} \frac{d a}{d t}, \\
\frac{1}{P_{\text {Rot }}} \frac{d P_{\text {Rot }}}{d t}=-\frac{1}{\Omega} \frac{d \Omega}{d t} .
\end{gathered}
$$

Where $P_{\text {orb }}$ denotes the orbital period and $P_{\text {Rot }}$ denotes the rotational period

Substituting the Equation (5) for $d e / d t$ into the following equation, we get the circurization scale time

$$
t_{\text {cir }}=\frac{e}{\frac{d e}{d t}}=\frac{t_{f}}{21 q(1+q) \lambda}\left(\frac{a}{R}\right)^{8} .
$$

In the following one uses the analytical method to solve the evolutional Equations (4)-(9) with the eccentricity $e$ as an independent variable

This paper considers the evolutional tendency of the orbit-spin of the synchronous binaries before the orbital circularization on the phase of the main sequence. We may assume that the radius, $R$, of the primary star may be regarded as no variation, i.e, $R$ is a constant on the phase of the main sequence star, but their separation or semi-major axis is variable due to the action of the tidal friction.

Combining the Equation (4) with (5), we obtain the differential equation

$$
\frac{1}{a} \frac{d a}{d e}=\frac{38}{7} e .
$$

Integrating this equation, we get

$$
a=a_{0} \exp \left[\frac{19}{7}\left(e^{2}-e_{0}^{2}\right)\right] .
$$

Substituting the Equation (12) into the Equation (5), we obtain the equation:

$$
\frac{1}{e} \frac{d e}{d t}=-21 q(1+q) \frac{\lambda}{t_{f}}\left(\frac{R}{a_{0}}\right)^{8} \exp \left[-\frac{152}{7}\left(e^{2}-e_{0}^{2}\right)\right] .
$$

By letting $c=152 / 7$, the differential Equation (13) can be written

$$
\frac{1}{e} \exp c\left(e^{2}-e_{0}^{2}\right) d e=\exp \left(-c e_{0}^{2}\right) \frac{\exp \left(c e^{2}\right)}{e} d e=-21 q(1+q) \frac{\lambda}{t_{f}}\left(\frac{R}{a_{0}}\right)^{8} d t .
$$

Using the expansion of series

$$
\exp \left(c e^{2}\right)=1+c e^{2}+\frac{1}{2} c^{2} e^{4}+\frac{1}{3} c^{3} e^{6}+\ldots \ldots
$$

Integrating the differential equation above, yields

$$
\exp \left(-c e_{0}^{2}\right)\left[\ln (e)+\frac{1}{2} c e^{2}+\frac{1}{8} c^{2} e^{4}+\ldots . . .\right]_{e_{0}}^{e}=-21 q(1+q) \frac{\lambda}{t_{f}}\left(\frac{R}{a_{0}}\right)^{8}\left(t-t_{0}\right) .
$$

We obtain time scale in terms of eccentricity, $e$, by neglecting the term with $e^{4}$

$$
t-t_{0}=-\frac{\ln \left(\frac{e}{e_{0}}\right)+\frac{1}{2} c\left(e^{2}-e_{0}^{2}\right)}{21 q(1+q) Q} .
$$

Here

$$
Q=\frac{\lambda}{t_{f}}\left(\frac{R}{a_{0}}\right)^{8} \exp \left(\frac{152}{7} e_{0}^{2}\right) .
$$

Combining the Equation (4) with (6), we get the equation

$$
a d a=-\frac{57}{18} \frac{(1+q)}{q}\left(\frac{I}{M}\right) \frac{d \Omega}{\Omega} .
$$


Integrating this equation, we obtain

$$
\Omega=\Omega_{0} \exp \left[-\frac{9}{57}\left(\frac{q}{1+q}\right)\left(\frac{M}{I}\right)\left(a^{2}-a_{0}^{2}\right)\right] .
$$

The from Equation (12) $a=a_{0} \exp \left[\frac{19}{7}\left(e^{2}-e_{0}^{2}\right)\right]$, so $a^{2}=a_{0}^{2} \exp \left[2 \times \frac{19}{7}\left(e^{2}-e_{0}^{2}\right)\right]$, hence

$$
a^{2}-a_{0}^{2}=a_{0}^{2}\left\{\exp \left[\frac{38}{7}\left(e^{2}-e_{0}^{2}\right)\right]-1\right\} .
$$

We obtain the variation of the angular velocity of primary in terms of eccentricity, $e$

$$
\Omega=\Omega_{0} \exp \left\{-\frac{9}{57}\left(\frac{q}{1+q}\right) \frac{M}{I} a_{0}^{2}\left\{\exp \left[\frac{38}{7}\left(a^{2}-a_{0}^{2}\right)\right]-1\right\}\right\} .
$$

The integrations of the Equations (7) - 9) can be obtained

$$
\begin{gathered}
\omega=\omega_{0} \exp \left[-\frac{57}{14}\left(e^{2}-e_{0}^{2}\right)\right], \\
P_{\text {orb }}=\left(P_{0}\right)_{\text {orb }} \exp \left[+\frac{57}{14}\left(e^{2}-e_{0}^{2}\right)\right], \\
P_{\text {Rot }}=\left(P_{0}\right)_{\text {Rot }} \exp \left\{+\frac{9}{57}\left(\frac{q}{1+q}\right) \frac{M}{I} a_{0}^{2}\left\{\exp \left[\frac{38}{7}\left(a^{2}-a_{0}^{2}\right)\right]-1\right\}\right\} .
\end{gathered}
$$

Next, one gives the analytical solutions of the secular evolutional equations with time $t$ as an independent variable on the main sequence.

For small eccentricity, $e$, such as Algol A, B, $e=0.015$, so $\frac{1}{2} c e^{2}=0.0024$, the second term of the right hand of formula (14) may be neglected., and then, we can obtain the eccentricity decreases with time from the formula (14)

$$
\begin{gathered}
e=e_{0} \exp \left[-21 q(1+q) Q\left(t-t_{0}\right)\right] . \\
e^{2}-e_{0}^{2}=e_{0}^{2}\left\{\exp \left[-42 q(1+q) Q\left(t-t_{0}\right)\right]-1\right\}
\end{gathered}
$$

Substituting Equation (22) or Equation (23) into Equation (12), we get

$$
\begin{gathered}
a=a_{0} \exp \left\{\frac{19}{7} e_{0}^{2}\left\{\exp \left[-42 q(1+q) Q\left(t-t_{0}\right)\right]-1\right\}\right\}, \\
a^{2}-a_{0}^{2}=a_{0}^{2}\left\{\exp \left\{\frac{38}{7} e_{0}^{2}\left\{\exp \left[-42 q(1+q) Q\left(t-t_{0}\right)\right]-1\right\}\right\}-1\right\} .
\end{gathered}
$$

Substituting the formula (22) into the formula (18) or the formula (25) into the formula (16), we obtain

$$
\Omega=\Omega_{0} \exp \left\{-\frac{9}{57} \frac{q}{(1+q)} \frac{M}{I} a_{0}^{2}\left\{\exp \left\{\frac{38}{7} e_{0}^{2}\left\{\exp \left[-42 q(1+q) Q\left(t-t_{0}\right)\right]-1\right\}\right\}-1\right\}\right\} .
$$

The integrations of the Equations (7) - 9) can be obtained

$$
\begin{gathered}
\omega=\omega_{0} \exp \left[-\frac{57}{14}\left(e^{2}-e_{0}^{2}\right)\right], \\
P_{\text {orb }}=\left(P_{0}\right)_{\text {orb }} \exp \left\{+\frac{57}{14} e_{0}\left\{\exp \left[-42 q(1+q) Q\left(t-t_{0}\right)\right]-1\right\}\right\}, \\
P_{\text {Rot }}=\left(P_{\text {Rot }}\right)_{0} \exp \left\{+\frac{9}{57} \frac{q}{(1+q)} \frac{M}{I} a_{0}^{2}\left\{\exp \left\{\frac{38}{7} e_{0}^{2}\left\{\exp \left[-42 q(1+q) Q\left(t-t_{0}\right)\right]-1\right\}\right\}-1\right\}\right\} .
\end{gathered}
$$




\section{THE ORBIT-SPIN EVOLUTION OF THE SYNCHRONOUS BINARIES (ALGOL A, B )}

The eclipsing binary system Algol ( $\beta$ Per) at least consists of three components A, B, C. In fact, there is also a massive unseen fourth component $\mathrm{D}$ (Hopkins 1976). Algol A (primary) is a main sequence star $\left(\mathrm{B}_{8} \cdot \mathrm{V}\right)$. Algol B (secondary) is a subgaint $\left(\mathrm{g} \mathrm{K}_{0}\right)$. The separation between $\mathrm{A}$ and $\mathrm{B}$ is very near mutually and the system Algol A, B is regarded as a synchronous binaries due to the action of the tidal friction. Based on Giuricin et al. (1984a) the mean rotational angular velocity of primary A is $v=56 \mathrm{~km} \mathrm{~s}^{-1}$, the mean orbital angular velocity (the synchronized velocity), $v_{k}=55 \mathrm{~km} \mathrm{~s}^{-1}$, and based on Tan (1985) gave $v_{\text {sini }}=55 \mathrm{~km} \mathrm{~s}^{-1}, v_{\mathrm{syn}}=55 \mathrm{~km} \mathrm{~s}^{-1}$. So A and B is a near synchronous binaries and based on apparent descriptive method for judging the synchronization of rotation of binary stars (Li 2004, 2009) Algol A, B is a near synchronization binaries. Hence this paper chooses Algol A, B as synchronous binaries to calculate the orbital circularization and evlution of the orbit and spin before circularization on the main sequence. For the data of Algol A, B, we cite the orbital period $\left(P_{0}\right)_{\text {orb }}=2.8672$ day, $a_{0}=14.03 R_{\odot}, M=3.7 M_{\odot}, M^{\prime}=0.81 M_{\odot}, R=2.74 R_{\odot}, R^{\prime}=3.60 R_{\odot}, q=M^{\prime} / M=0.22$, $T_{e}=12010^{\circ} \mathrm{K}$ (Brancewicz \& Dworak 1980). $e_{0}=0.015$ (Tomkin \& Lambert 1978, Harrington 1984), $\Omega_{0}=v / R=2.9365 \times 10^{-5} \mathrm{rad} \mathrm{s}^{-1}$ and $t_{f}=t_{f \odot}\left(M / M_{\odot}\right)^{1 / 3}\left(T_{e} / e \odot\right)^{-4 / 3}, t_{f \odot}=0.433 \mathrm{yr}$, $T_{e \odot}=5770^{0} \mathrm{~K}, t_{f}=0.2519 \mathrm{yr}, L=2.2 L_{\odot}($ Popper 1980$)$.

Where $T_{e}$ is the effective temperature (Zahn \& Bouchet 1989)

Recently, Yang et al. (2011) present XMM-Newton observation of the eclipsing binary Algol. Their results of research are valuable.

As Zahn \& Bouchet (1989) showed that when the coefficient $\lambda^{l m}$ are all equal, then

$$
\lambda=k_{2} \quad \text { (the apsidal motion constant). }
$$

$\lambda=k_{2}$ (the apsidal motiom constant) is calculated from the formula given by Cowling (1938) and letting $k_{1}=k_{2}=k$

$$
k=\frac{P_{\mathrm{orb}} / P^{\prime}}{\left(\frac{R}{a}\right)^{5}\left(1+16 \frac{M^{\prime}}{M}\right)+\left(\frac{R^{\prime}}{a}\right)^{5}\left(1+16\left(\frac{M}{M^{\prime}}\right)\right.} .
$$

Here $P^{\prime}$ denotes the period of advance of the apsital line, $P^{\prime}=2.476$ year for Algol A, B given by Hegedüs (1988). Substituting $P_{\text {orb }}, P^{\prime}, a, M, M^{\prime}, R$ and $R^{\prime}$ into the above formula, we get

$$
\lambda=k=0.003308 .
$$

The moment of inertia $I$

$$
I=K M R^{2} .
$$

$K$ is culculated from the formula $\frac{1}{K}=\frac{3}{2}\left(n+\frac{5}{2}\right)$ Schatzman 1963), The polytropic index $n=3$ for the main sequence star (Algol A). So $K=4 / 33=0.1212$.

$$
\frac{M}{I}=\frac{M}{K M R^{2}}=\frac{1}{K R^{2}}=0.02268 \times 10^{-10} \mathrm{~km}^{-2}, \quad \exp \left(152 \mathrm{e}_{0}^{2} / 7\right)=1.00489 .
$$

Substituting the values of $k, t_{f}, R, a$ and $\exp \left(152 e_{0}^{2} / 7\right)$ into the formula (15), we obtain

$$
Q=2.7 \times 10^{-8} \mathrm{yr}^{-1} .
$$

Let us estimate the numerical solutions when the orbit of Algol A, B achieves the circularizatuion

At first, we evaluate the circularization time scale Substituting the values of $q, R_{0}, a_{0}, t_{f}$ and $\lambda=k=0.003308$ into the formula $[10$, we obtain the circularization time scale

$$
t_{\text {cir }}=6.4589 \times 10^{6} \mathrm{yr} .
$$

Next we estimate the numerical solution of evolutionary tendency of the orbit-spin when Algol A, B achieved the orbital circularization time. By letting the intial time $t_{0}=0$, and substituting $t_{\text {cir }}=6.4589 \mathrm{yr}$ into the formulas (22), 24) or (16)-29], we get $a=14.0226 R_{\odot}, e=0,0056$, 
$\omega=2.1931 \mathrm{rad} \mathrm{d}^{-1}, P_{\text {orb }}=2.8649 \mathrm{~d}, \Omega=2.5536 \mathrm{rad} \mathrm{d}^{-1}, P_{\text {Rot }}=2.4605 \mathrm{~d} ; \delta a=-0.0074 R_{\odot}$, $\delta e=-0.0094, \delta \omega=+0.0017 \mathrm{rad} \mathrm{d}^{-1}, \delta P_{\mathrm{orb}}=-0.0023 \mathrm{~d}, \delta \Omega=+0.0156 \mathrm{rad} \mathrm{d}^{-1}, \delta P_{\mathrm{Rot}}=$ $-0.0160 \mathrm{~d}$.

The life time (age) is based on stellar mass-lose $\dot{M}$, i.e

$$
t_{\text {life }}=\frac{M}{\dot{M}} .
$$

The value of its may be calculated from the formule given by Bowers \& Deeming (1984)

$$
\frac{d\left(M / M_{\Theta}\right)}{d t}=3 \times 10^{-8} \frac{\left(R / R_{\Theta}\right)\left(L / L_{\Theta}\right)}{\left(M / M_{\Theta}\right)}\left(M_{\Theta} / \mathrm{yr}\right)
$$

or calculated from the formula given by Nieuwenhuijzen \& de Jager (1990)

$$
\log \dot{M}=-14.02+1.24 \log \left(L / L_{\Theta}\right)+0.81 \log \left(R / R_{\Theta}\right)+0.16 \log \left(M / M_{\Theta}\right)
$$

Substituting these datd of $M, R$ and $L$ into the formula $(36)$ and $(35)$, we get the life time (age)

$$
t_{\text {life }}=7.5703 \times 10^{7} \mathrm{yr}(\text { age }) .
$$

The lime time for the speed up of spin

$$
t_{\Omega}=\frac{\Omega}{\dot{\Omega}}=t_{f} / 36 q^{2}\left(\frac{M}{I}\right) k e^{2} a^{2}\left(\frac{R}{a}\right)^{8}=4.2447 \times 10^{8} \mathrm{yr} .
$$

The orbital spiral time (the collasped time of system)

$$
t_{a}=\frac{a}{\dot{a}}=t_{f} / 114 q(1+q) k e^{2}(R / a)^{8}=5.2273 \times 10^{9} \mathrm{yr} .
$$

\section{DICUSSION AND CONCLUSIONS}

(1) The set of the Equations (4)-6 fits in synchronous binaries on the pre-main sequence, main sequence and post-main sequence phase according to the radii of a stars. The raduis of the late type star is variable due to the gravitational contraction on the pre-main sequence phase. The raduis of gaint star is variable possibly due to the expansion of shell on the post-main sequence phase. On the main sequence phase the raduis of star is stable. Its raduis can be regarded as constant. These refer to the raduis of primary star because in the Equations (4) -6 $R$ denotes the raduis of the primary star. It does not refer to the gaint star.

(2) The research of this paper differs from that of Zahn \& Bouchet (1989) in some aspects. Zahn \& Boucher's paper researched the orbital evolution and circularization of binary stars on pre-main sequence phase by analytical method and for non-synchronized equations by the method of numerical integration. In the analytical method the radii of binaries are variable due to gravitational contraction, but the semi-major axis is not variable including the main sequence phase. However the present paper studies the orbit-spin of binary stars on the main sequence phase by analytical method in which the star's raduis is not variable and the semi-major axis is variable due to tidal friction. In Zahn \& Boucher's paper they must use the method of numerical integration to solve non-synchronized equations. However in the present paper the author may use the analytical method to solve the synchronized equations. Zahn \& Boucher estimate that the eccentricity decreases from 0.005 to 0.0043 in 10 billion for binaries $0.5 M_{\odot}+0.5 M_{\odot}$ on the main sequence phase. The present paper estimates that the eccentricity decreases from 0.015 to 0.0056 in 6.45 mega for binaries $3.07 M_{\odot}+0.81 M_{\odot}$ on the main sequence phase. Hence both results are different.

(3) The results of the solution for integrating diferential equations by analytical mathod are some less different with that by the method of numerical integration. For example, the semi-major axis $a=$ $14.0226 R_{\odot}$ for Algol A, B when the circularization time $\left(6.4589 \times 10^{6} \mathrm{yr}\right)$ by the former method and $a=14.0126 R_{\odot}$ by the latter method. However this difference is very small. 
(4) The circularization time occurs before the life time (age), and the lime time for the speed up of spin and the spiral time (the collasped time of the system) occur after the life time (age). Hence the latter both are meaningless.

(5) In the system of Algol A, B, C the tidal friction in triple stars (Kiseleva et al. 1998) and the perturbing effect of the third star (Algol C) ( $\mathrm{Li}$ 2006) may decircularize the orbit of the second star (Algol B). In this paper one does not consider these effect.

One obtained the following conclusions:

(1) The eccentricity decreases gradually with time until the orbital circularization, i.e until $e$ decreases to the circularization time.

(2) The semi-major axis shrinks gradually with time or with eccentricity decreases.

(3) The orbital period shortens gradually with time or with circularization.

(4) The rotational angular velocity of primary component speeds up with time gradually.

\section{References}

Alexander, M. E. 1973, Ap\&SS, 23, 459

Bowers, R. L., \& Deeming, T. 1984, Astrophysics. Vol. I: Stars. Vol. II: Interstellar matter and galaxies. eds. R. L. Bowers, T. D. Jones, \& Bartlett Publishers, Inc., 20 Park Plaza, Boston, Mass

Brancewicz, H. K., \& Dworak, T. Z. 1980, Acta Astronomica, 30, 501

Cowling, T. G. 1938, MNRAS, 98, 734

Darwin, G. H. 1879, Philosophical Transactions of the Royal Society of London, 170, 1

Giuricin, G., Mardirossian, F., \& Mezzetti, M. 1984a, A\&A, 131, 152

Giuricin, G., Mardirossian, F., \& Mezzetti, M. 1984b, A\&A, 135, 393

Harrington, R. S. 1984, ApJ, 277, L69

Hegedüs, T. 1988, Bulletin d'Information du Centre de Donnees Stellaires, 35, 15

Hopkins, J. 1976, Glossary of astronomy and astrophysics (University of Chicago press), 3

Huang, R., \& Zeng, Y. 2000, Science in China A: Mathematics, 43, 331

Hut, P. 1980, A\&A, 92, 167

Hut, P. 1981, A\&A, 99, 126

Keppens, R., Solanki, S. K., \& Charbonnel, C. 2000, A\&A, 359, 552

Kiseleva, L. G., Eggleton, P. P., \& Mikkola, S. 1998, MNRAS, 300, 292

Li, L. 1998, Acta Astrophysica Sinica, 18, 77

Li, L.-S. 2004, Journal of Astrophysics and Astronomy, 25, 203, Erratum, 2005, 26, 447

Li, L.-S. 2006, AJ, 131, 994

Li, L. S. 2009, Astronomical Research \& Technology (Publ. Nat. Astron. Obs. China), 6, 264

Meibom, S., Mathieu, R. D., \& Stassun, K. 2005, Bulletin of the American Astronomical Society, 36, \#107.02

Meibom, S., Mathieu, R. D., \& Stassun, K. G. 2006, ApJ, 653, 621

Nieuwenhuijzen, H., \& de Jager, C. 1990, A\&A, 231, 134

Pan, K. K. 1996, Acta Astrophysica Sinica, 16, 370

Popper, D. M. 1980, ARA\&A, 18, 115

Rajamohan, R., \& Venkatakrishnan, P. 1981, Bulletin of the Astronomical Society of India, 9, 309

Schatzman, E. 1963, in proceedings of the XXVIIIth Course of the International School of Physics "Enrico Fermi”,

Star Evolution, ed. L. Gratton (New York: Academic Press), 177

Tan, H.-S. 1985, Acta Astronomica Sinica, 26, 226

Tomkin, J., \& Lambert, D. L. 1978, ApJ, 222, L119

Tassoul, J.-L. 1987, ApJ, 322, 856

Yang, X.-J., Lu, F.-J., Aschenbach, B., \& Chen, L. 2011, Research in Astron. Astrophys. (RAA), 11, 457

Zahn, J.-P. 1965, Compt. Rend. Acad. Sci. Paris, 260, 413

Zahn, J.-P. 1966a, Ann. Astrophy., 29, 313 
Zahn, J. P. 1966b, Annales d'Astrophysique, 29, 489

Zahn, J. P. 1966c, Annales d'Astrophysique, 29, 565

Zahn, J.-P. 1975, A\&A, 41, 329

Zahn, J.-P. 1977, A\&A, 57, 383

Zahn, J.-R. 1978, A\&A, 67, 162

Zahn, J.-P. 1989, A\&A, 220, 112

Zahn, J.-P., \& Bouchet, L. 1989, A\&A, 223, 112 